\title{
Density and viscosity calculation of a quaternary system of amine absorbent before and after carbon dioxide absorption
}

Khuyen Viet Bao Tran, Miho Sato, Keiichi Yanase, Tsuyoshi Yamaguchi, Hiroshi

$$
\text { Machida }{ }^{*} \text {, and Koyo Norinaga }
$$

Graduate School of Engineering, Nagoya University, Furocho, Chikusaku, Nagoya, 464-8603, Japan

*corresponding author: Hiroshi MACHIDA

E-mail: machida.hiroshi@material.nagoya-u.ac.jp 


\section{Experimental densities:}

Table S1. Densities $\rho$ of calibration solutions EAE/DEGDEE/water mixtures at $p=101300 \mathrm{~Pa}$. $w_{\mathrm{i}}$ stands for weight fraction of component

$i^{\text {a }}$

\begin{tabular}{|c|c|c|c|c|c|c|c|c|c|}
\hline \multirow{2}{*}{$w_{\text {EAE }}$} & \multirow{2}{*}{$w_{\mathrm{H} 2 \mathrm{O}}$} & \multicolumn{8}{|c|}{$\rho / \mathrm{kg} \cdot \mathrm{m}^{-3}$} \\
\hline & & $293.15 \mathrm{~K}$ & $303.15 \mathrm{~K}$ & $313.15 \mathrm{~K}$ & $323.15 \mathrm{~K}$ & 333.15 & $343.15 \mathrm{~K}$ & 353.15 & $363.15 \mathrm{~K}$ \\
\hline 0.200 & 0.800 & 995.83 & 991.58 & 986.75 & 981.28 & & & & \\
\hline 0.400 & 0.600 & 993.48 & 986.60 & 979.59 & 972.34 & 964.80 & 956.82 & 948.74 & \\
\hline 0.600 & 0.400 & 979.77 & 972.04 & 963.99 & 955.94 & 947.65 & 939.16 & 930.48 & 921.54 \\
\hline 0.800 & 0.200 & 954.39 & 946.43 & 938.70 & 930.46 & 922.15 & 913.64 & 904.91 & 895.89 \\
\hline 1.000 & 0.000 & 916.21 & 907.89 & 899.87 & 891.78 & 883.52 & 875.24 & 866.78 & 858.16 \\
\hline 0.200 & 0.600 & 996.08 & 988.19 & 980.80 & 972.65 & 964.44 & 956.09 & 947.34 & 938.41 \\
\hline 0.400 & 0.400 & 980.61 & 972.12 & 963.82 & 955.11 & 946.29 & 937.35 & 928.27 & 918.68 \\
\hline 0.600 & 0.200 & 953.57 & 945.15 & 936.79 & 928.22 & 919.38 & 910.61 & 901.47 & 892.07 \\
\hline
\end{tabular}




$\begin{array}{llllllllll}0.800 & 0.000 & 914.32 & 905.68 & 897.26 & 888.40 & 880.32 & 871.65 & 862.88 & 853.91 \\ 0.200 & 0.400 & 980.24 & 971.12 & 962.09 & 952.79 & 943.40 & 933.86 & 924.14 & 914.25 \\ 0.400 & 0.200 & 952.31 & 943.30 & 934.43 & 925.46 & 916.33 & 907.05 & 897.57 & 887.91 \\ 0.600 & 0.000 & 912.08 & 903.17 & 894.43 & 885.60 & 876.70 & 867.71 & 858.61 & 849.28 \\ 0.200 & 0.200 & 950.45 & 941.17 & 932.02 & 922.62 & 913.07 & 903.24 & 893.43 & 883.35 \\ 0.400 & 0.000 & 910.08 & 900.78 & 891.69 & 882.52 & 873.26 & 863.89 & 854.46 & 844.88 \\ 0.200 & 0.000 & 908.47 & 898.85 & 889.41 & 879.88 & 870.29 & 860.68 & 851.00 & 841.22 \\ 0.000 & 0.000 & 906.85 & 896.96 & 887.42 & 877.70 & 867.98 & 858.24 & 848.44 & 838.57\end{array}$

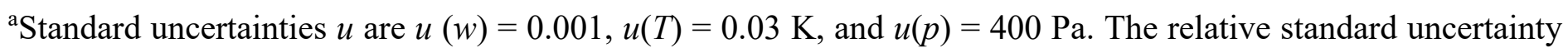
is $u_{\mathrm{r}}(\rho)=0.002$.

Table S2. Densities $\rho$ of calibration solutions EAE/water/carbamate mixtures at $p=101300 \mathrm{~Pa} . w_{\mathrm{i}}$ stands for weight fraction of component

$i^{\text {a }}$

\begin{tabular}{|c|c|c|c|c|c|c|}
\hline \multirow{2}{*}{$w_{\mathrm{EAE}}$} & \multirow{2}{*}{$w_{\text {Carbamate }}$} & \multicolumn{5}{|c|}{$\rho / \mathrm{kg} \cdot \mathrm{m}^{-3}$} \\
\hline & & $293.15 \mathrm{~K} \quad 303.15 \mathrm{~K} \quad 313.15 \mathrm{~K}$ & $323.15 \mathrm{~K}$ & $343.15 \mathrm{~K}$ & 353.15 & $363.15 \mathrm{~K}$ \\
\hline
\end{tabular}




$\begin{array}{lllllllllll}0.7624 & 0.2376 & 0.10 & 961.71 & 953.82 & 945.96 & 938.03 & 930.00 & 921.88 & 913.38 & 904.82 \\ 0.5461 & 0.4539 & 0.20 & 1003.8 & 996.16 & 988.55 & 980.86 & 973.07 & 965.18 & 957.09 & 948.68 \\ 0.3484 & 0.6516 & 0.30 & 1043.4 & 1036.1 & 1028.8 & 1021.4 & 1013.8 & 1006.2 & 998.21 \\ 0.1670 & 0.8329 & 0.40 & 1080.4 & 1073.0 & 1066.1 & 1058.8 & 1051.4 & 1043.8 & \\ 0.9000 & 0.0000 & 0.00 & 937.99 & 929.80 & 921.91 & 913.87 & 905.66 & 897.30 & 888.72 & 880.08 \\ 0.6894 & 0.2149 & 0.10 & 978.83 & 971.25 & 963.25 & 955.53 & 947.76 & 939.86 & 931.57 & 922.88 \\ 0.4959 & 0.4122 & 0.20 & 1017.2 & 1009.8 & 1002.5 & 994.96 & 987.30 & 979.51 & 971.51 & 963.26 \\ 0.3177 & 0.5941 & 0.30 & 1053.6 & 1046.5 & 1039.3 & 1032.1 & 1024.7 & 1017.1 & 1009.3 & \\ 0.1528 & 0.7622 & 0.40 & 1087.2 & 1080.3 & 1073.4 & 1066.3 & 1059.1 & 1051.6 & & 935.23 \\ 0.5980 & 0.2103 & 0.11 & 991.40 & 983.67 & 976.23 & 968.46 & 960.30 & 952.14 & 943.79 & 935.23 \\ 0.4449 & 0.3698 & 0.20 & 1025.5 & 1018.1 & 1010.8 & 1003.3 & 995.40 & 987.58 & 979.50 & 971.22 \\ 0.2861 & 0.5351 & 0.30 & 1058.2 & 1051.1 & 1044.0 & 1036.7 & 1029.2 & 1021.6 & 1013.5 & \end{array}$

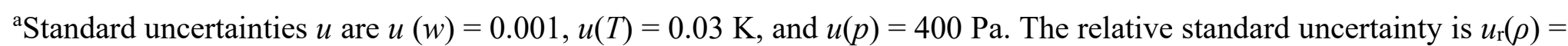
0.002 . 
Table S3. Densities $\rho$ of calibration solutions EAE/DEGDEE/carbamate mixtures at $p=101300 \mathrm{~Pa}$. $w_{\mathrm{i}}$ stands for weight fraction of component $i .^{\text {a }}$

\begin{tabular}{|c|c|c|c|c|c|c|c|c|c|c|}
\hline \multirow{2}{*}{$w_{\mathrm{EAE}}$} & \multirow{2}{*}{$w_{\text {Carbamate }}$} & \multirow{2}{*}{$\begin{array}{c}\alpha / \\
\mathrm{mol} \cdot \mathrm{mol}^{-1}\end{array}$} & \multicolumn{8}{|c|}{$\rho / \mathrm{kg} \cdot \mathrm{m}^{-3}$} \\
\hline & & & $293.15 \mathrm{~K}$ & $303.15 \mathrm{~K}$ & $313.15 \mathrm{~K}$ & $323.15 \mathrm{~K}$ & 333.15 & $343.15 \mathrm{~K}$ & 353.15 & $363.15 \mathrm{~K}$ \\
\hline 0.3000 & 0.0000 & 0.00 & 908.79 & 899.24 & 889.9200 & 880.65 & 871.18 & 861.69 & 852.12 & 842.44 \\
\hline 0.2808 & 0.0223 & 0.03 & 911.78 & 902.41 & 893.00 & 883.62 & 874.20 & 864.68 & 855.01 & 845.10 \\
\hline 0.2240 & 0.0882 & 0.12 & 924.06 & 914.57 & 905.24 & 895.88 & 886.39 & 876.68 & 866.77 & \\
\hline 0.1688 & 0.1524 & 0.21 & 936.54 & 927.07 & 917.82 & 908.44 & 898.82 & 888.76 & & \\
\hline 0.1446 & 0.1803 & 0.25 & 939.98 & 930.50 & 921.26 & 911.85 & 902.16 & 891.96 & & \\
\hline 0.5000 & 0.0000 & 0.00 & 910.67 & 901.46 & 892.58 & 883.60 & 874.49 & 865.32 & 855.97 & 846.54 \\
\hline 0.4665 & 0.0371 & 0.03 & 916.63 & 907.53 & 898.64 & 889.65 & 880.55 & 871.33 & 862.02 & 852.41 \\
\hline 0.3480 & 0.1687 & 0.14 & 941.56 & 932.43 & 923.62 & 914.68 & 905.61 & 896.37 & 886.82 & 876.75 \\
\hline 0.2255 & 0.3046 & 0.26 & 967.09 & 958.10 & 949.35 & 940.49 & 931.42 & 922.05 & & \\
\hline
\end{tabular}




$\begin{array}{llllllllllll}0.1765 & 0.3590 & 0.31 & 973.49 & 964.58 & 955.86 & 946.97 & 937.85 & 928.33 & & \\ 0.7000 & 0.0000 & 0.00 & 912.62 & 903.91 & 895.37 & 886.73 & 877.99 & 869.17 & 860.21 & 851.04 \\ 0.6352 & 0.0689 & 0.04 & 922.50 & 913.79 & 905.24 & 896.62 & 887.88 & 879.06 & 870.07 & 860.90 \\ 0.4364 & 0.2803 & 0.17 & 962.77 & 954.21 & 945.84 & 937.35 & 928.74 & 919.99 & 910.99 & 901.55 \\ 0.2403 & 0.4887 & 0.31 & 1005.4 & 996.89 & 988.69 & 980.35 & 971.88 & 963.21 & 954.03 & \\ 0.1873 & 0.5450 & 0.35 & 1016.3 & 1008.2 & 1000.1 & 991.81 & 983.29 & 974.43 & \end{array}$

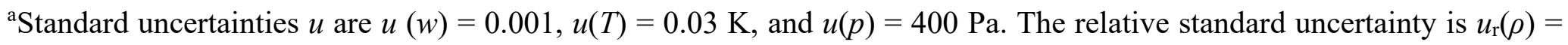
0.002 .

Table S4. Densities $\rho$ of calculation solutions at $p=101300 \mathrm{~Pa} . w_{\mathrm{i}}$ stands for weight fraction of component $i^{\text {a }}$

\begin{tabular}{|c|c|c|c|c|c|c|c|c|c|c|c|}
\hline sample & $w_{\mathrm{EAE}}$ & $w_{\mathrm{H} 2 \mathrm{O}}$ & $w_{\text {Carbamate }}$ & \multicolumn{8}{|c|}{$\rho / \mathrm{kg} \cdot \mathrm{m}^{-3}$} \\
\hline A1/ Lean (0.183) & 0.010 & 0.000 & 0.007 & 911.00 & 901.09 & 891.25 & 881.39 & 871.50 & 861.62 & 851.67 & 841.64 \\
\hline
\end{tabular}




\begin{tabular}{|c|c|c|c|c|c|c|c|c|c|c|c|}
\hline A2/ Lean (0.246) & 0.026 & 0.004 & 0.031 & 913.76 & 903.90 & 894.21 & 884.38 & 874.36 & 864.32 & 854.17 & 843.95 \\
\hline A2/ Rich (0.398) & 0.126 & 0.176 & 0.607 & 1064.5 & 1057.2 & 1049.8 & 1042.2 & 1034.5 & 1026.2 & 1016.0 & \\
\hline A3/ Lean (0.139) & 0.074 & 0.018 & 0.036 & 918.07 & 908.36 & 898.73 & 889.05 & 879.24 & 869.24 & 859.01 & \\
\hline A3/ Rich (0.284) & 0.252 & 0.176 & 0.414 & 1025.9 & 1018.2 & 1010.4 & 1002.5 & 994.21 & 986.13 & 977.45 & \\
\hline A4/ Lean (0.098) & 0.191 & 0.056 & 0.058 & 934.17 & 924.86 & 915.66 & 906.36 & 896.88 & 887.32 & 877.40 & 867.04 \\
\hline A4/ Rich (0.156) & 0.317 & 0.140 & 0.179 & 973.05 & 964.49 & 955.99 & 947.34 & 938.50 & 929.55 & 920.34 & 910.81 \\
\hline $\mathrm{A} 5 / \mathrm{x}(0.07)$ & 0.281 & 0.007 & 0.057 & 922.46 & 913.03 & 903.83 & 894.56 & 885.19 & 875.63 & 865.83 & 855.59 \\
\hline \multicolumn{12}{|l|}{$30 / 50 / 20(\alpha)$} \\
\hline B6/ Lean (0.065) & 0.042 & 0.031 & 0.008 & 914.16 & 904.16 & 894.33 & 884.45 & 874.44 & 864.44 & 854.38 & 844.28 \\
\hline B6/ Rich (0.391) & 0.124 & 0.261 & 0.556 & 1082.2 & 1074.9 & 1067.6 & 1060.1 & 1052.4 & 1044.5 & & \\
\hline B7/ Lean (0.249) & 0.020 & 0.000 & 0.025 & 914.21 & 904.34 & 894.57 & 884.71 & 874.75 & 864.76 & 854.71 & 844.58 \\
\hline B7/ Rich (0.388) & 0.130 & 0.262 & 0.555 & 1071.0 & 1063.7 & 1056.2 & 1048.6 & 1040.9 & 1033.0 & 1024.9 & \\
\hline B8/ Lean (0.076) & 0.126 & 0.089 & 0.028 & 932.22 & 922.65 & 913.28 & 903.83 & 894.18 & 884.39 & 874.37 & \\
\hline B8/ Rich (0.144) & 0.303 & 0.228 & 0.152 & 981.05 & 972.33 & 963.94 & 955.10 & 946.23 & 937.25 & 928.03 & 918.53 \\
\hline
\end{tabular}




\begin{tabular}{|c|c|c|c|c|c|c|c|c|c|c|c|}
\hline \multicolumn{12}{|l|}{$30 / 40 / 30(\alpha)$} \\
\hline C9/ Lean (0.331) & 0.008 & 0.024 & 0.018 & 917.33 & 907.52 & 897.70 & 887.84 & 877.90 & 867.89 & 857.80 & 847.65 \\
\hline C9/ Rich (0.399) & 0.094 & 0.377 & 0.461 & 1074.7 & 1067.1 & 1059.6 & 1052.0 & 1044.2 & 1036.3 & 1028.2 & \\
\hline C10/ Lean (0.086) & 0.110 & 0.103 & 0.029 & 926.41 & 916.61 & 907.01 & 897.34 & 887.51 & 877.52 & 867.32 & \\
\hline C10/ Rich (0.224) & 0.207 & 0.297 & 0.209 & 1013.3 & 1004.7 & 996.44 & 988.02 & 979.42 & 970.66 & 961.71 & 952.56 \\
\hline \multicolumn{12}{|l|}{$30 / 70(\alpha)$} \\
\hline D11/ Lean (0.158) & 0.019 & 0.000 & 0.011 & & & 885.67 & 877.50 & 868.42 & & & \\
\hline D11/ Rich (0.491) & 0.013 & 0.000 & 0.913 & 1088.6 & 1080.7 & 1072.8 & & & & & \\
\hline $\mathrm{D} 12 / \mathrm{x}(0.117)$ & 0.224 & $0.008^{\mathrm{b}}$ & 0.085 & 927.05 & 917.55 & 908.23 & 898.87 & 889.35 & 879.61 & 869.32 & \\
\hline D13/ x (0.049) & 0.262 & $0.025^{\mathrm{b}}$ & 0.036 & & 907.66 & 898.28 & 888.92 & 879.47 & 869.86 & 860.02 & 849.92 \\
\hline \multicolumn{12}{|c|}{$\begin{array}{l}\mathrm{CO}_{2} \text {-unloaded } \\
\text { (EAE/DEGDEE/Water) }\end{array}$} \\
\hline $40 / 60 / 0$ & 0.400 & 0.000 & 0.000 & 909.52 & 900.09 & 890.93 & 881.78 & 872.53 & 863.17 & 853.73 & 844.18 \\
\hline $30 / 60 / 10$ & 0.300 & 0.100 & 0.000 & 931.60 & 922.33 & 913.23 & 903.99 & 894.61 & 885.10 & 875.31 & 865.49 \\
\hline $30 / 50 / 20$ & 0.300 & 0.200 & 0.000 & 952.75 & 943.44 & 934.12 & 924.65 & 914.86 & 904.62 & 896.79 & 886.87 \\
\hline
\end{tabular}




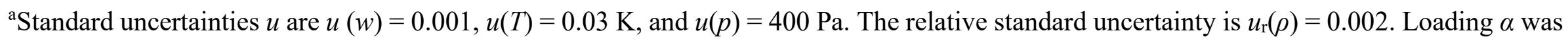
defined as the moles of $\mathrm{CO}_{2}$ absorbed into solutions $/$ moles of EAE, $\mathrm{mol} \cdot \mathrm{mol}^{-1}$.

${ }^{\mathrm{b}}$ As chemicals were used as received; water partly exists even in those solutions without adding water on sample preparation.

\section{Experimental viscosities:}

Table S5. Viscosity $\eta$ of calibration solutions EAE/DEGDEE/water mixtures at $p=101300 \mathrm{~Pa} . w_{\mathrm{i}}$ stands for weight fraction of component $i^{\text {a }}$

\begin{tabular}{cccccccccc}
\multirow{2}{*}{$w_{\text {EAE }}$} & $w_{\mathrm{H} 2 \mathrm{O}}$ & \multicolumn{8}{c}{$\eta / \mathrm{mPa} \cdot \mathrm{s}$} \\
\cline { 3 - 10 } & & $293.15 \mathrm{~K}$ & $303.15 \mathrm{~K}$ & $313.15 \mathrm{~K}$ & $323.15 \mathrm{~K}$ & 333.15 & $343.15 \mathrm{~K}$ & 353.15 & $363.15 \mathrm{~K}$ \\
\hline 0.200 & 0.800 & 2.7228 & 1.9344 & 1.4216 & 1.0927 & & & & \\
0.400 & 0.600 & 7.3148 & 4.6173 & 3.1217 & 2.2245 & 1.6520 & 1.2642 & 1.0191 & \\
0.600 & 0.400 & 17.554 & 10.325 & 6.5442 & 4.3900 & 3.1061 & 2.2916 & 1.7374 & 1.3615 \\
0.800 & 0.200 & 31.752 & 17.939 & 10.853 & 6.9768 & 4.7241 & 3.3401 & 2.4472 & 1.8511
\end{tabular}




$\begin{array}{llllllllll}1.000 & 0.000 & 14.541 & 9.2659 & 6.2191 & 4.3797 & 3.2068 & 2.4192 & 1.8731 & 1.4833 \\ 0.200 & 0.600 & 5.6046 & 3.6874 & 2.5751 & 1.8889 & 1.4397 & 1.1327 & 0.91540 & 0.74330 \\ 0.400 & 0.400 & 11.023 & 6.8557 & 4.5431 & 3.1792 & 2.3234 & 1.7572 & 1.3700 & 1.0964 \\ 0.600 & 0.200 & 16.373 & 9.9630 & 6.4398 & 4.3919 & 3.1382 & 2.3124 & 1.7603 & 1.3763 \\ 0.800 & 0.000 & 7.4820 & 5.1757 & 3.7175 & 2.7641 & 2.1779 & 1.6651 & 1.3346 & 1.0903 \\ 0.200 & 0.400 & 7.1030 & 4.6589 & 3.2272 & 2.3500 & 1.7759 & 1.3880 & 1.1149 & 0.91560 \\ 0.400 & 0.200 & 9.0175 & 5.8949 & 4.0596 & 2.9205 & 2.1793 & 1.6764 & 1.3246 & 1.0717 \\ 0.600 & 0.000 & 4.2755 & 3.1365 & 2.3720 & 1.8440 & 1.4661 & 1.1894 & 0.98264 & 0.82670 \\ 0.200 & 0.200 & 5.1565 & 3.6043 & 2.6247 & 1.9799 & 1.5399 & 1.2315 & 1.0027 & 0.83471 \\ 0.400 & 0.000 & 2.6009 & 2.0071 & 1.5837 & 1.2761 & 1.0482 & 0.87616 & 0.74284 & 0.63985 \\ 0.200 & 0.000 & 1.7399 & 1.3966 & 1.1396 & 0.94626 & 0.79820 & 0.68213 & 0.59186 & 0.52068 \\ 0.000 & 0.000 & 1.3045 & 1.0796 & 0.90507 & 0.77010 & 0.66266 & 0.57726 & 0.50948 & 0.45589\end{array}$

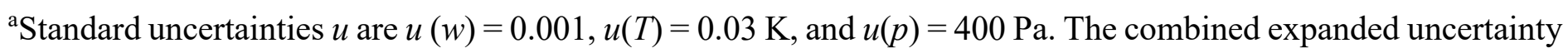
at 0.95 level of confidence $(k=2)$ is $U(\eta)=0.10 \eta$. 
Table S6. Viscosities $\eta$ of calibration solutions EAE/water/carbamate mixtures at $p=101300 \mathrm{~Pa}$. $w_{\mathrm{i}}$ stands for weight fraction of component $i .^{\text {a }}$

\begin{tabular}{|c|c|c|c|c|c|c|c|c|c|c|}
\hline \multirow{2}{*}{$w_{\mathrm{EAE}}$} & \multirow{2}{*}{$w_{\text {Carbamate }}$} & \multirow{2}{*}{$\begin{array}{c}\alpha / \\
\mathrm{mol} \cdot \mathrm{mol}^{-1}\end{array}$} & \multicolumn{8}{|c|}{$\eta / \mathrm{mPa} \cdot \mathrm{s}$} \\
\hline & & & $293.15 \mathrm{~K}$ & $303.15 \mathrm{~K}$ & $313.15 \mathrm{~K}$ & $323.15 \mathrm{~K}$ & 333.15 & $343.15 \mathrm{~K}$ & 353.15 & $363.15 \mathrm{~K}$ \\
\hline 0.7624 & 0.2376 & 0.10 & 51.764 & 29.140 & 17.595 & 11.317 & 7.6565 & 5.4015 & 3.9491 & 2.9728 \\
\hline 0.5461 & 0.4539 & 0.20 & 197.64 & 99.532 & 54.380 & 31.959 & 19.966 & 13.144 & 9.0276 & 6.4029 \\
\hline 0.3484 & 0.6516 & 0.30 & 791.41 & 360.96 & 179.92 & 97.291 & 56.310 & 34.530 & 22.199 & \\
\hline 0.1670 & 0.8329 & 0.40 & 3083.1 & 1280.1 & 585.81 & 291.97 & 155.61 & 87.235 & & \\
\hline 0.9000 & 0.0000 & 0.00 & 30.012 & 17.301 & 10.602 & 6.8824 & 4.6919 & 3.3430 & 2.4597 & 1.8589 \\
\hline 0.6894 & 0.2149 & 0.10 & 74.107 & 39.545 & 22.855 & 14.068 & 9.1671 & 6.2711 & 4.4680 & 3.2933 \\
\hline 0.4959 & 0.4122 & 0.20 & 188.28 & 93.814 & 50.753 & 29.571 & 18.395 & 12.080 & 8.3012 & 5.9197 \\
\hline 0.3177 & 0.5941 & 0.30 & 495.86 & 229.86 & 116.88 & 64.445 & 38.105 & 23.914 & 15.830 & \\
\hline 0.1528 & 0.7622 & 0.40 & 1282.9 & 560.37 & 269.64 & 141.32 & 79.716 & 47.748 & & \\
\hline
\end{tabular}




$\begin{array}{lllllllllll}0.5980 & 0.2103 & 0.11 & 60.064 & 32.365 & 18.826 & 11.710 & 7.7080 & 5.3325 & 3.8348 & 2.8471 \\ 0.4449 & 0.3698 & 0.20 & 112.63 & 58.349 & 32.679 & 19.675 & 12.601 & 8.4883 & 5.9718 & 4.3643 \\ 0.2861 & 0.5351 & 0.30 & 217.09 & 107.54 & 57.968 & 33.700 & 20.905 & 13.684 & 9.3350 \\ 0.1382 & 0.6891 & 0.40 & 420.29 & 199.14 & 103.16 & 58.064 & 35.024 & 22.382 & 14.736\end{array}$

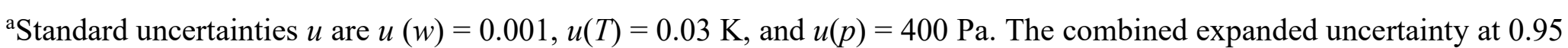
level of confidence $(k=2)$ is $U(\eta)=0.10 \eta$.

Table S7. Viscosities $\eta$ of calibration solutions EAE/DEGDEE/carbamate mixtures at $p=101300 \mathrm{~Pa}$. $w_{\mathrm{i}}$ stands for weight fraction of component $i .^{\text {a }}$

\begin{tabular}{|c|c|c|c|c|c|c|c|c|c|c|}
\hline \multirow{2}{*}{$w_{\mathrm{EAE}}$} & \multirow{2}{*}{$w_{\text {Carbamate }}$} & \multirow{2}{*}{$\begin{array}{c}\alpha / \\
\mathrm{mol} \cdot \mathrm{mol}^{-1}\end{array}$} & \multicolumn{8}{|c|}{$\eta / \mathrm{mPa} \cdot \mathrm{s}$} \\
\hline & & & $293.15 \mathrm{~K}$ & $303.15 \mathrm{~K}$ & $313.15 \mathrm{~K}$ & $323.15 \mathrm{~K}$ & 333.15 & $343.15 \mathrm{~K}$ & 353.15 & $363.15 \mathrm{~K}$ \\
\hline 0.3000 & 0.0000 & 0.00 & 2.0278 & 1.6039 & 1.2910 & 1.0559 & 0.87864 & 0.74166 & 0.63514 & 0.55239 \\
\hline 0.2808 & 0.0223 & 0.03 & 2.1377 & 1.6815 & 1.3495 & 1.1010 & 0.91264 & 0.76630 & 0.65222 & 0.55429 \\
\hline 0.2240 & 0.0882 & 0.12 & 2.6937 & 2.1112 & 1.6698 & 1.3450 & 1.1010 & 0.91417 & 0.73514 & \\
\hline 0.1688 & 0.1524 & 0.21 & 3.7609 & 2.8178 & 2.1675 & 1.7077 & 1.3686 & 1.1081 & & \\
\hline
\end{tabular}




$\begin{array}{lllllllllllll}0.1446 & 0.1803 & 0.25 & 4.3240 & 3.1601 & 2.3969 & 1.8698 & 1.4859 & 1.1950 & \\ 0.5000 & 0.0000 & 0.00 & 3.2001 & 2.4218 & 1.8670 & 1.4781 & 1.1937 & 0.98081 & 0.82026 & 0.69679 \\ 0.4665 & 0.0371 & 0.03 & 3.6282 & 2.7070 & 2.0741 & 1.6301 & 1.3089 & 1.0712 & 0.89097 & 0.75326 \\ 0.3480 & 0.1687 & 0.14 & 6.5053 & 4.6247 & 3.3965 & 2.5764 & 2.0051 & 1.5933 & 1.2857 & 1.0141 \\ 0.2255 & 0.3046 & 0.26 & 14.845 & 9.6387 & 6.5942 & 4.7078 & 3.4716 & 2.6278 & & \\ 0.1765 & 0.3590 & 0.31 & 20.216 & 12.377 & 8.1813 & 5.7093 & 4.1391 & 3.0800 & & \\ 0.7000 & 0.0000 & 0.00 & 5.4809 & 3.9024 & 2.8795 & 2.1888 & 1.7065 & 1.3589 & 1.1033 & 0.91285 \\ 0.6352 & 0.0689 & 0.04 & 6.8314 & 4.7701 & 3.4571 & 2.5909 & 1.9964 & 1.5738 & 1.2662 & 1.0372 \\ 0.4364 & 0.2803 & 0.17 & 20.113 & 12.702 & 8.4546 & 5.9033 & 4.2866 & 3.2101 & 2.4628 & 1.9247 \\ 0.2403 & 0.4887 & 0.31 & 85.005 & 46.959 & 27.915 & 17.661 & 11.747 & 8.1108 & 5.5889 & \end{array}$

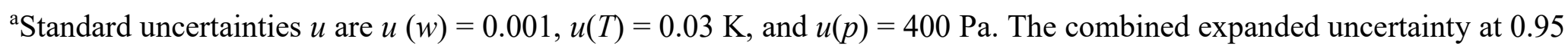
level of confidence $(k=2)$ is $U(\eta)=0.10 \eta$. 
Table S8. Viscosities $\eta$ of calculation solutions at $p=101300 \mathrm{~Pa} . w_{\mathrm{i}}$ stands for weight fraction of component $i^{\text {a }}$

\begin{tabular}{|c|c|c|c|c|c|c|c|c|c|c|c|}
\hline \multirow[b]{2}{*}{ sample } & \multirow[b]{2}{*}{$w_{\mathrm{EAE}}$} & \multirow[b]{2}{*}{$w_{\mathrm{H} 2 \mathrm{O}}$} & \multirow[b]{2}{*}{$w_{\text {Carbamate }}$} & \multicolumn{8}{|c|}{$\eta / \mathrm{mPa} \cdot \mathrm{s}$} \\
\hline & & & & $293.15 \mathrm{~K}$ & $303.15 \mathrm{~K}$ & $313.15 \mathrm{~K}$ & $323.15 \mathrm{~K}$ & 333.15 & $343.15 \mathrm{~K}$ & 353.15 & $363.15 \mathrm{~K}$ \\
\hline $30 / 60 / 10(\alpha)$ & & & & & & & & & & & \\
\hline A1/ Lean (0.183) & 0.010 & 0.000 & 0.007 & 1.4188 & 1.1594 & 0.96186 & 0.80896 & 0.69204 & 0.59758 & 0.52492 & 0.46610 \\
\hline A1/ Rich (0.472) & 0.035 & 0.185 & 0.728 & 279.6 & 136.79 & 73.345 & 42.645 & 26.499 & 17.396 & & \\
\hline A2/ Lean (0.246) & 0.026 & 0.004 & 0.031 & 1.5608 & 1.2696 & 1.0440 & 0.87144 & 0.73836 & 0.63292 & 0.55054 & 0.48747 \\
\hline A2/ Rich (0.398) & 0.126 & 0.176 & 0.607 & 138.76 & 71.402 & 40.173 & 24.353 & 15.720 & 10.669 & 7.4892 & \\
\hline A3/ Lean (0.139) & 0.074 & 0.018 & 0.036 & 1.9071 & 1.5079 & 1.2175 & 1.0007 & 0.83502 & 0.70693 & 0.60919 & \\
\hline A3/ Rich (0.284) & 0.252 & 0.176 & 0.414 & 58.701 & 32.326 & 19.363 & 12.386 & 8.3667 & 5.9073 & 4.3339 & \\
\hline A4/ Lean (0.098) & 0.191 & 0.056 & 0.058 & 3.8586 & 2.808 & 2.1195 & 1.6478 & 1.3129 & 1.0655 & 0.88065 & 0.74275 \\
\hline A4/ Rich (0.156) & 0.317 & 0.140 & 0.179 & 15.766 & 9.8157 & 6.5137 & 4.5548 & 3.3209 & 2.5013 & 1.9401 & 1.5397 \\
\hline $\mathrm{A} 5 / \mathrm{x}(0.07)$ & 0.281 & 0.007 & 0.057 & 3.1066 & 2.3703 & 1.8598 & 1.4971 & 1.2306 & 1.0295 & 0.87369 & 0.75084 \\
\hline
\end{tabular}




\begin{tabular}{|c|c|c|c|c|c|c|c|c|c|c|c|}
\hline B6/ Lean (0.065) & 0.042 & 0.031 & 0.008 & 1.5314 & 1.2453 & 1.0290 & 0.86120 & 0.73162 & 0.62923 & 0.54741 & 0.45867 \\
\hline B6/ Rich (0.391) & 0.124 & 0.261 & 0.556 & 92.079 & 49.582 & 28.905 & 18.077 & 11.959 & 8.3046 & & \\
\hline B7/ Lean (0.249) & 0.020 & 0.000 & 0.025 & 1.5794 & 1.2829 & 1.0580 & 0.88635 & 0.75348 & 0.64815 & 0.56667 & 0.50505 \\
\hline B7/ Rich (0.388) & 0.130 & 0.262 & 0.555 & 77.954 & 42.468 & 25.088 & 15.847 & 10.626 & 7.4689 & 5.4468 & \\
\hline B8/ Lean (0.076) & 0.126 & 0.089 & 0.028 & 3.1434 & 2.3569 & 1.8173 & 1.4398 & 1.1657 & 0.97283 & 0.81396 & \\
\hline B8/ Rich (0.144) & 0.303 & 0.228 & 0.152 & 14.594 & 9.2413 & 6.1733 & 4.3416 & 3.1812 & 2.4070 & 1.8730 & 1.4944 \\
\hline \multicolumn{12}{|l|}{$30 / 40 / 30(\alpha)$} \\
\hline C9/ Lean (0.331) & 0.008 & 0.024 & 0.018 & 1.6815 & 1.3552 & 1.1103 & 0.92400 & 0.78008 & 0.66933 & 0.58413 & 0.49555 \\
\hline C9/ Rich (0.399) & 0.094 & 0.377 & 0.461 & 35.497 & 20.650 & 12.980 & 8.7056 & 6.1255 & 4.4789 & 3.3875 & \\
\hline C10/ Lean (0.086) & 0.110 & 0.103 & 0.029 & 2.2549 & 1.7553 & 1.3939 & 1.1297 & 0.93174 & 0.78112 & 0.66524 & \\
\hline C10/ Rich (0.224) & 0.207 & 0.297 & 0.209 & 16.981 & 10.506 & 6.9008 & 4.8110 & 3.4929 & 2.6289 & 2.0418 & 1.6118 \\
\hline \multicolumn{12}{|l|}{$30 / 70(\alpha)$} \\
\hline D11/ Lean (0.158) & 0.019 & 0.000 & 0.011 & & & 0.95974 & 0.81123 & 0.68663 & & & \\
\hline D11/ Rich (0.491) & 0.013 & 0.000 & 0.913 & 3116.8 & 1234.4 & 549.43 & & & & & \\
\hline
\end{tabular}




\begin{tabular}{|c|c|c|c|c|c|c|c|c|c|c|c|}
\hline $\mathrm{D} 12 / \mathrm{x}(0.117)$ & 0.224 & $0.008^{\mathrm{b}}$ & 0.085 & 3.0183 & 2.3221 & 1.8371 & 1.4887 & 1.2281 & 1.0259 & 0.86780 & \\
\hline $\mathrm{D} 13 / \mathrm{x}(0.049)$ & 0.262 & $0.025^{\mathrm{b}}$ & 0.036 & & 1.9234 & 1.5432 & 1.2653 & 1.0571 & 0.89793 & 0.76995 & 0.64258 \\
\hline \multicolumn{12}{|c|}{$\begin{array}{l}\mathrm{CO}_{2} \text {-unloaded } \\
\text { (EAE/DEGDEE/Water) }\end{array}$} \\
\hline $40 / 60 / 0$ & 0.400 & 0.000 & 0.000 & 2.6029 & 2.0268 & 1.6158 & 1.3170 & 1.0944 & 0.9257 & 0.79441 & 0.69093 \\
\hline $30 / 60 / 10$ & 0.300 & 0.100 & 0.000 & 4.3083 & 3.0990 & 2.3029 & 1.7647 & 1.3865 & 1.1135 & 0.91335 & 0.76327 \\
\hline $30 / 50 / 20$ & 0.300 & 0.200 & 0.000 & 6.5599 & 4.4616 & 3.1668 & 2.3360 & 1.7791 & 1.3919 & 1.1163 & 0.91526 \\
\hline $30 / 40 / 30$ & 0.300 & 0.300 & 0.000 & 8.4852 & 5.5184 & 3.7953 & 2.7312 & 2.0416 & 1.5761 & 1.2506 & 1.0106 \\
\hline
\end{tabular}

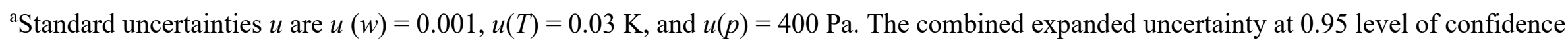
$(k=2)$ is $U(\eta)=0.10 \eta$. Loadings $\alpha$ was defined as the moles of $\mathrm{CO}_{2}$ absorbed into solutions $/ \mathrm{moles}$ of EAE, $\mathrm{mol} \cdot \mathrm{mol}^{-1}$.

${ }^{\mathrm{b}}$ As chemicals were used as received; water partly exists even in those solutions without adding water on sample preparation. 


\section{Densities and viscosities of pure EAE and DEGDEE}

\section{II.1. Pure EAE}

Table S9. Densities $\rho$ /viscosities $\eta$ of pure EAE at different temperatures. ${ }^{\text {a }}$

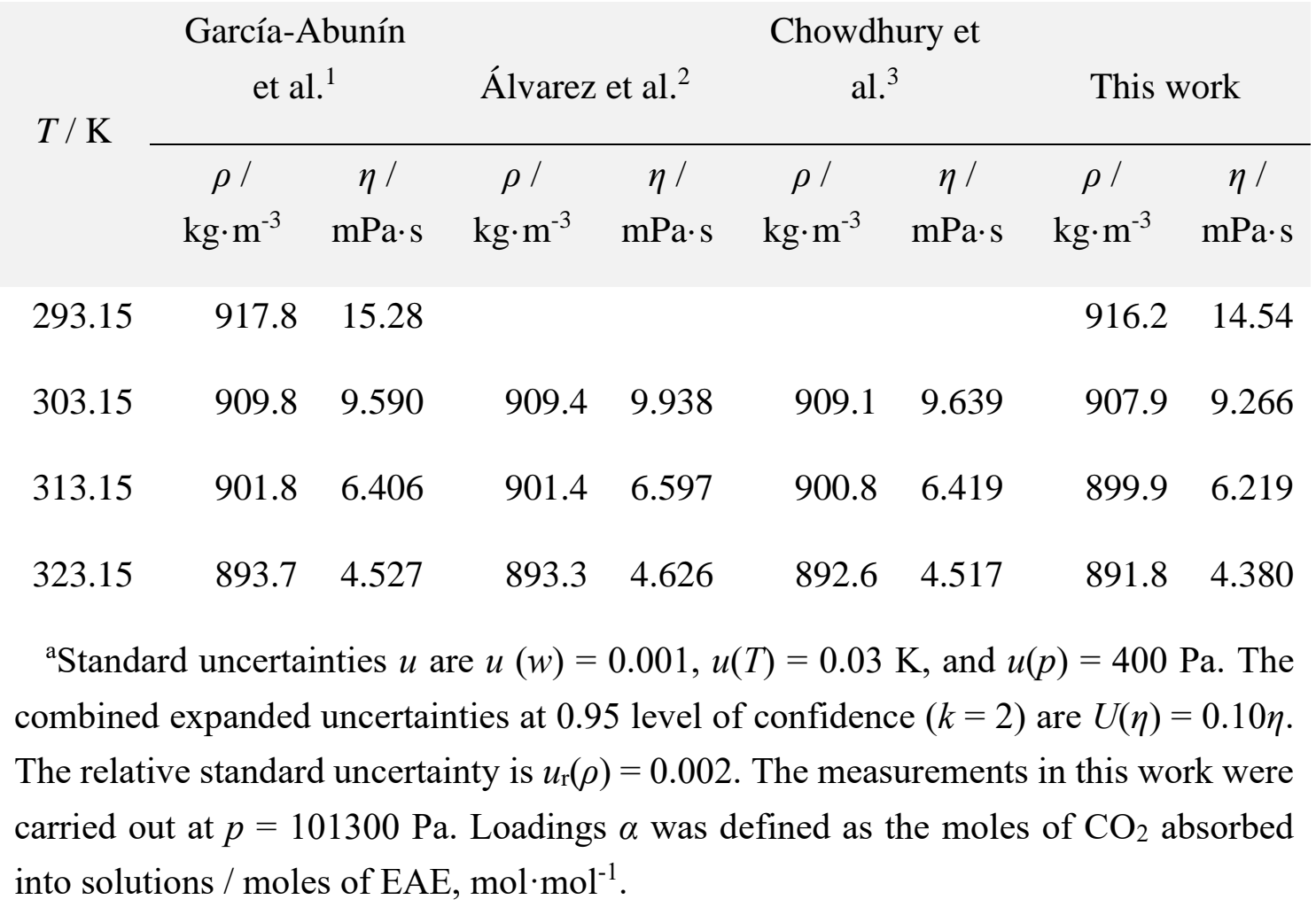




\section{II.2. Pure DEGDEE}

Table S10. Densities $\rho$ /viscosities $\eta$ of pure DEGDEE at different temperatures. ${ }^{\text {a }}$

\begin{tabular}{|c|c|c|c|c|c|c|}
\hline \multirow[b]{2}{*}{$T / \mathrm{K}$} & \multicolumn{2}{|c|}{$\mathrm{Ku}$ and $\mathrm{Tu}^{4}$} & \multicolumn{2}{|c|}{ Esteve et al. ${ }^{5}$} & \multicolumn{2}{|c|}{ this work } \\
\hline & $\begin{array}{c}\rho / \\
\mathrm{kg} \cdot \mathrm{m}^{-3}\end{array}$ & $\begin{array}{c}\eta / \\
\mathrm{mPa} \cdot \mathrm{s}\end{array}$ & $\begin{array}{c}\rho / \\
\mathrm{kg} \cdot \mathrm{m}^{-3}\end{array}$ & $\begin{array}{c}\eta / \\
\mathrm{mPa} \cdot \mathrm{s}\end{array}$ & $\begin{array}{c}\rho / \\
\mathrm{kg} \cdot \mathrm{m}^{-3}\end{array}$ & $\begin{array}{c}\eta / \\
\mathrm{mPa} \cdot \mathrm{s}\end{array}$ \\
\hline 293.15 & 906.8 & 1.358 & 907.9 & 1.364 & 906.9 & 1.305 \\
\hline 303.15 & 897.3 & 1.134 & 898.4 & 1.140 & 897.0 & 1.080 \\
\hline 313.15 & 887.6 & 0.996 & 889.0 & 0.971 & 887.4 & 0.905 \\
\hline 323.15 & 878.0 & 0.837 & 879.5 & 0.840 & 877.7 & 0.770 \\
\hline 333.15 & 868.2 & 0.732 & 870.0 & 0.737 & 868.0 & 0.663 \\
\hline 343.15 & 858.4 & 0.648 & 860.2 & 0.654 & 858.2 & 0.577 \\
\hline 353.15 & & & 850.0 & 0.587 & 848.4 & 0.509 \\
\hline 363.15 & & & 840.2 & 0.532 & 838.6 & 0.456 \\
\hline
\end{tabular}

a Standard uncertainties $u$ are $u(w)=0.001, u(T)=0.03 \mathrm{~K}$, and $u(p)$

$=400 \mathrm{~Pa}$. The combined expanded uncertainties at 0.95 level of confidence $(k=2)$ are $U(\eta)=0.10 \eta$. The relative standard uncertainty is $u_{\mathrm{r}}(\rho)=0.002$. The measurements in this work were carried out at $p$ $=101300 \mathrm{~Pa}$. Loadings $\alpha$ was defined as the moles of $\mathrm{CO}_{2}$ absorbed into solutions $/$ moles of EAE, $\mathrm{mol} \cdot \mathrm{mol}^{-1}$. 


\section{Extrapolation of densities and viscosities for "pure" EAE carbamate}

Because pure EAE carbamate doesn't exist by itself, its densities and viscosities were estimated from densities and viscosities of pure $\mathrm{EAE}$ at various $\mathrm{CO}_{2}$ loadings. We blew $\mathrm{CO}_{2}$ to pure EAE to full loading. This solution was then adjusted to loadings of $0.1,0.2,0.3$, and $0.4 \mathrm{~mol} \cdot \mathrm{mol}^{-1}$. Carbamate is formed by the reaction of $2 \mathrm{~mol}$ of EAE and $1 \mathrm{~mol}$ of $\mathrm{CO}_{2}$; therefore, we assumed that product of pure EAE at loading 0.5 would be the same condition of pure EAE carbamate. We measured prepared $\mathrm{CO}_{2}-$ loaded pure EAE solutions at various temperatures, and extrapolated the viscosity and density curve to obtain the value of pure
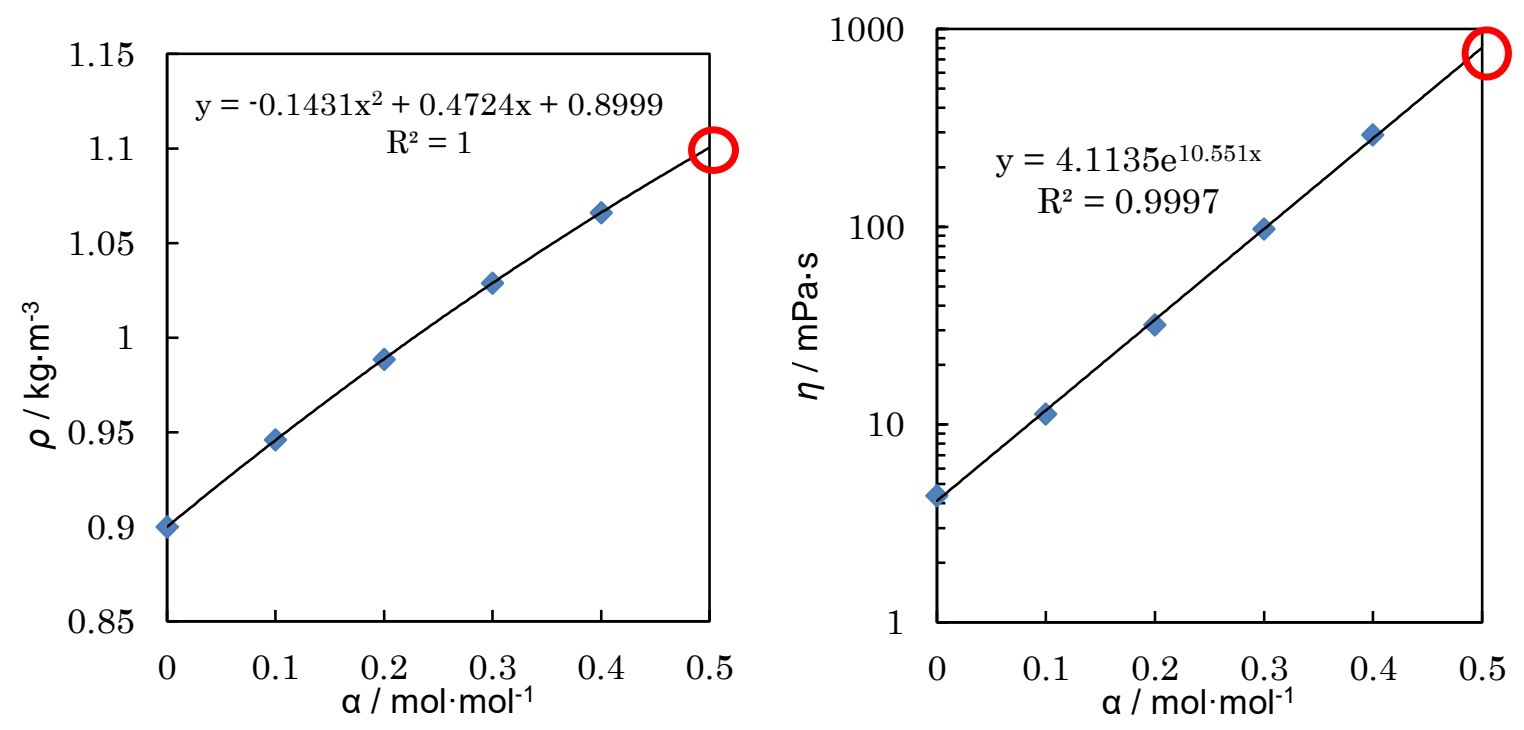

Figure S1. Extrapolation for pure EAE carbamate at $313 \mathrm{~K}$. Marks and solid lines: experimental data and fitted curves. The red circles are the obtained values of densities and viscosities of pure EAE carbamate at this temperature. 
EAE at loading 0.5 . Figure $\mathrm{S} 1$ shows how extrapolation was performed at $313.15 \mathrm{~K}$. Density has a polynomial correlation to the loading. ${ }^{6}$ The logarithm of viscosity and loading has a linear relation. ${ }^{7}$ The red circles are the density and viscosity of EAE carbamate at $313.15 \mathrm{~K}$.

Table S11. Densities $\rho$ of samples used in the extrapolation of densities for "pure" EAE

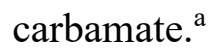

\begin{tabular}{clllll}
\multicolumn{1}{c}{$\alpha /$} & \multicolumn{5}{c}{$\rho / \mathrm{kg} \cdot \mathrm{m}^{-3}$} \\
$\mathrm{~mol} \cdot \mathrm{mol}^{-1}$ & 0.00 & 0.10 & 0.20 & 0.30 & 0.40 \\
\cline { 2 - 6 } $293.15 \mathrm{~K}$ & 916.21 & 961.71 & 1003.78 & 1043.43 & 1080.37 \\
$303.15 \mathrm{~K}$ & 907.89 & 953.82 & 996.16 & 1036.13 & 1073.02 \\
$313.15 \mathrm{~K}$ & 899.87 & 945.96 & 988.55 & 1028.81 & 1066.05 \\
$323.15 \mathrm{~K}$ & 891.78 & 938.03 & 980.86 & 1021.38 & 1058.83 \\
$333.15 \mathrm{~K}$ & 883.52 & 930.00 & 973.07 & 1013.84 & 1051.44 \\
$343.15 \mathrm{~K}$ & 875.24 & 921.88 & 965.18 & 1006.15 & 1043.80 \\
$353.15 \mathrm{~K}$ & 866.78 & 913.38 & 957.09 & 998.21 & \\
$363.15 \mathrm{~K}$ & 858.16 & 904.82 & 948.68 & &
\end{tabular}

aStandard uncertainties $u$ are $u(w)=0.001, u(T)=0.03 \mathrm{~K}$, and $u(p)=400 \mathrm{~Pa}$. The relative standard uncertainty is $u_{\mathrm{r}}(\rho)=0.002$. The measurements in this work were carried out at $p=101300 \mathrm{~Pa}$. Loadings $\alpha$ was defined as the moles of $\mathrm{CO}_{2}$ absorbed into solutions / moles of EAE, $\mathrm{mol} \cdot \mathrm{mol}^{-1}$. 


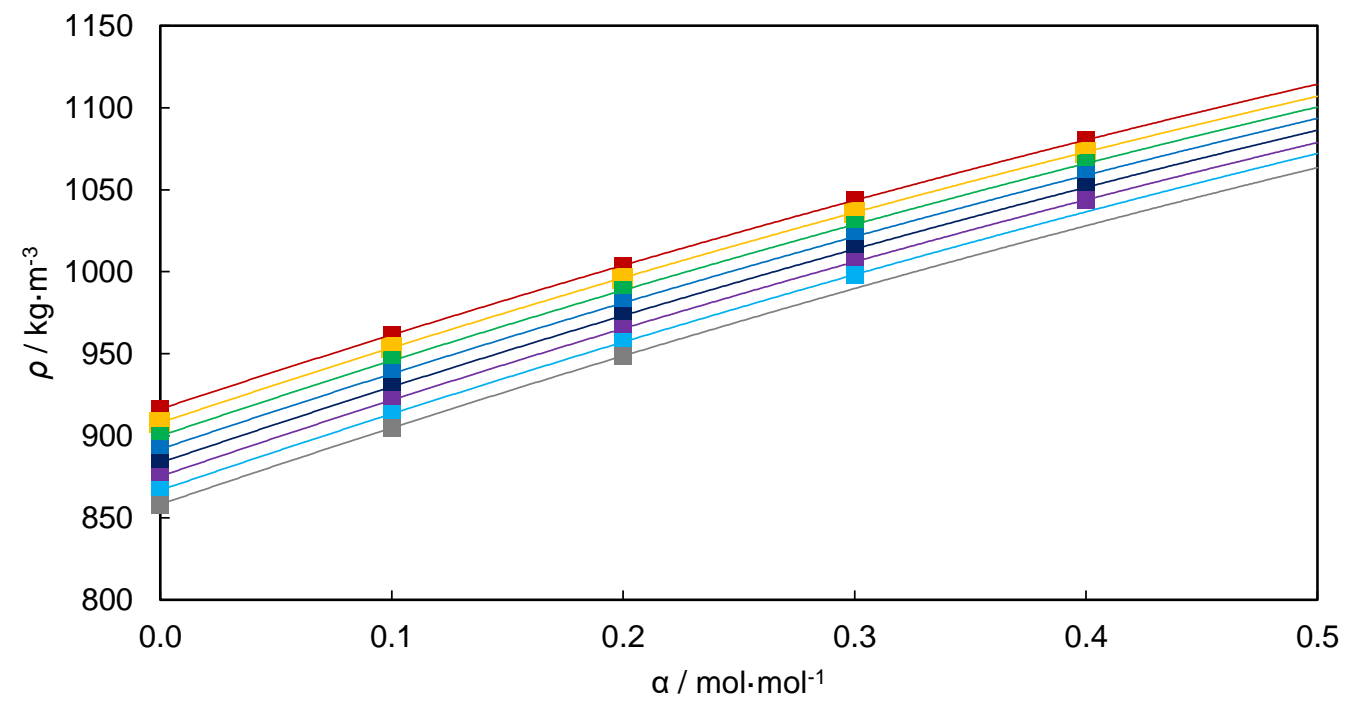

Figure S2. Density extrapolation for "pure" EAE carbamate data. $293.15 \mathrm{~K}$ (dark red), 303.15

K (orange), 313.15 K (green), 323.15 K (blue), 333.15 K (dark blue), $343.15 \mathrm{~K}$ (purple), 353.15 K (light blue) and 363.15 K(grey).

Table S12. Viscosities $\eta$ of samples used in the extrapolation of viscosities for "pure" EAE carbamate. $^{\mathrm{a}}$

\begin{tabular}{clllll}
\multicolumn{1}{c}{$\alpha$} & \multicolumn{5}{c}{$\eta / \mathrm{mPa} \cdot \mathrm{s}$} \\
$\mathrm{mol} \cdot \mathrm{mol}^{-1}$ & 0.00 & 0.10 & 0.20 & 0.30 & 0.40 \\
\cline { 2 - 6 } & 14.541 & 51.764 & 197.64 & 791.41 & 3083.1 \\
$303.15 \mathrm{~K}$ & 9.2659 & 29.140 & 99.532 & 360.96 & 1280.1 \\
$313.15 \mathrm{~K}$ & 6.2191 & 17.595 & 54.380 & 179.92 & 585.81 \\
$323.15 \mathrm{~K}$ & 4.3797 & 11.317 & 31.959 & 97.291 & 291.97 \\
$333.15 \mathrm{~K}$ & 3.2068 & 7.6565 & 19.966 & 56.310 & 155.61 \\
$343.15 \mathrm{~K}$ & 2.4192 & 5.4015 & 13.144 & 34.530 & 87.235
\end{tabular}




$\begin{array}{lllll}353.15 \mathrm{~K} & 1.8731 & 3.9491 & 9.0276 & 22.199 \\ 363.15 \mathrm{~K} & 1.4833 & 2.9728 & 6.4029 & \end{array}$

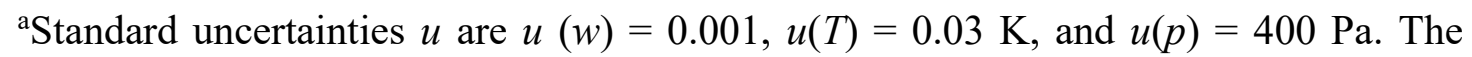
combined expanded uncertainty at 0.95 level of confidence $(k=2)$ is $U(\eta)=0.10 \eta$. The measurements in this work were carried out at $p=101300 \mathrm{~Pa}$. Loadings $\alpha$ was defined as the moles of $\mathrm{CO}_{2}$ absorbed into solutions / moles of EAE, $\mathrm{mol} \cdot \mathrm{mol}^{-1}$.

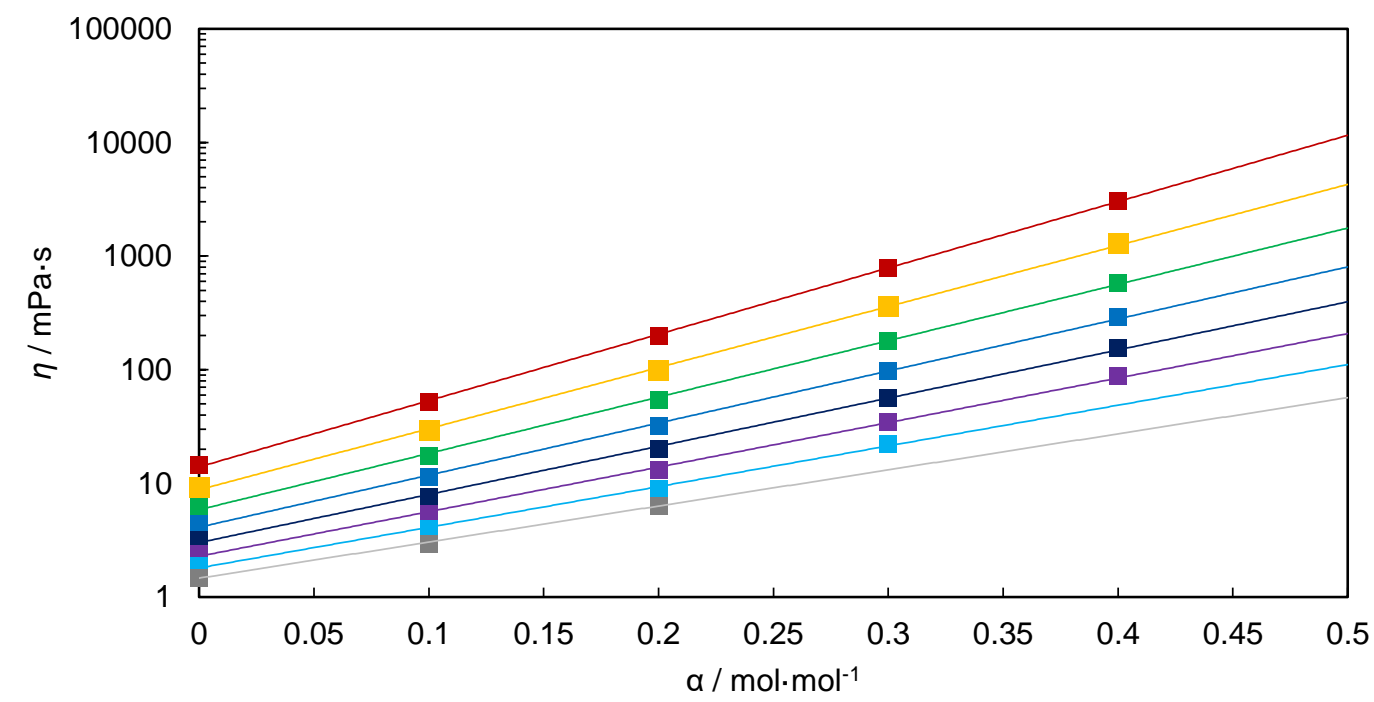

Figure S3. Viscosity extrapolation for "pure" EAE carbamate data. 293.15 K (dark red), 303.15

K (orange), 313.15 K (green), 323.15 K (blue), 333.15 K (dark blue), 343.15 K (purple), 353.15

K (light blue) and $363.15 \mathrm{~K}$ (grey). 


\section{Density and viscosity models for pure components}

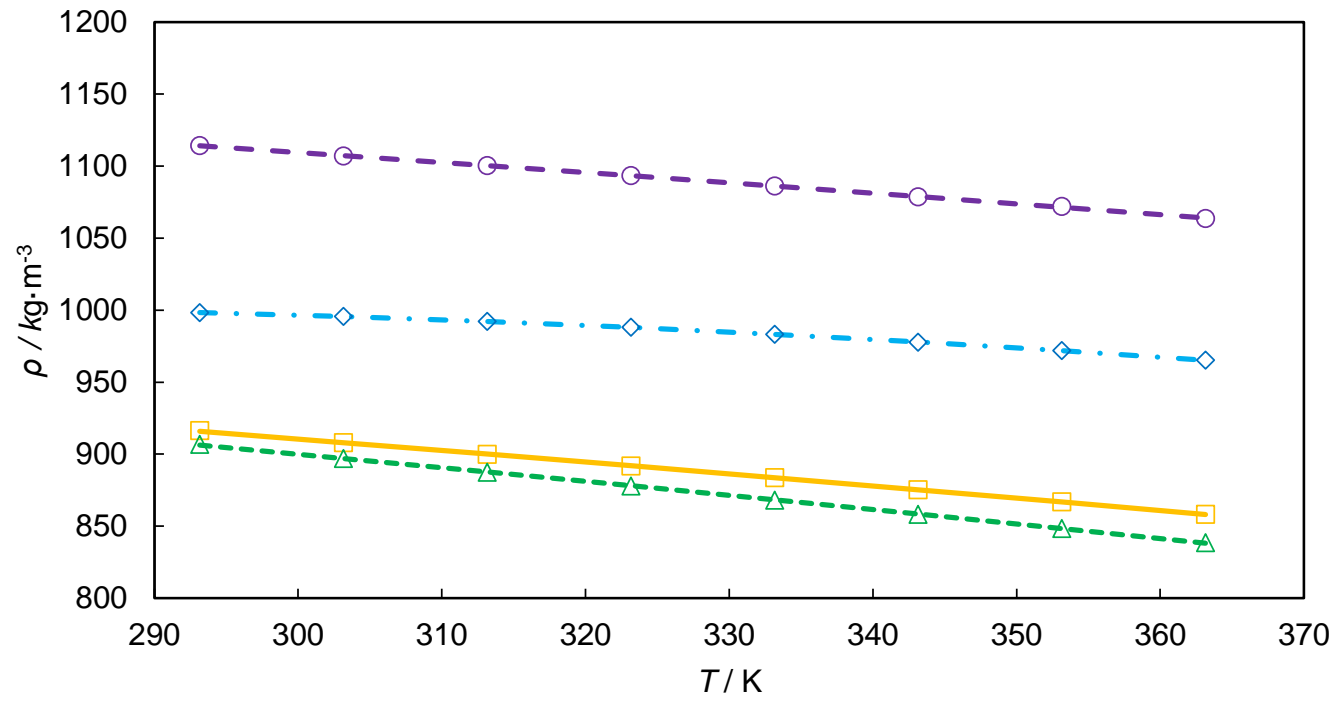

Figure S4. Experimental and calculated densities of pure components (marks and lines:

experimental and calculated data): EAE ( $\square$ and solid line), DEGDEE ( $\Delta$ and square-dot line), carbamate ( $\circ$ and dashed line), and water ( $\diamond$ and dash-dot line). 


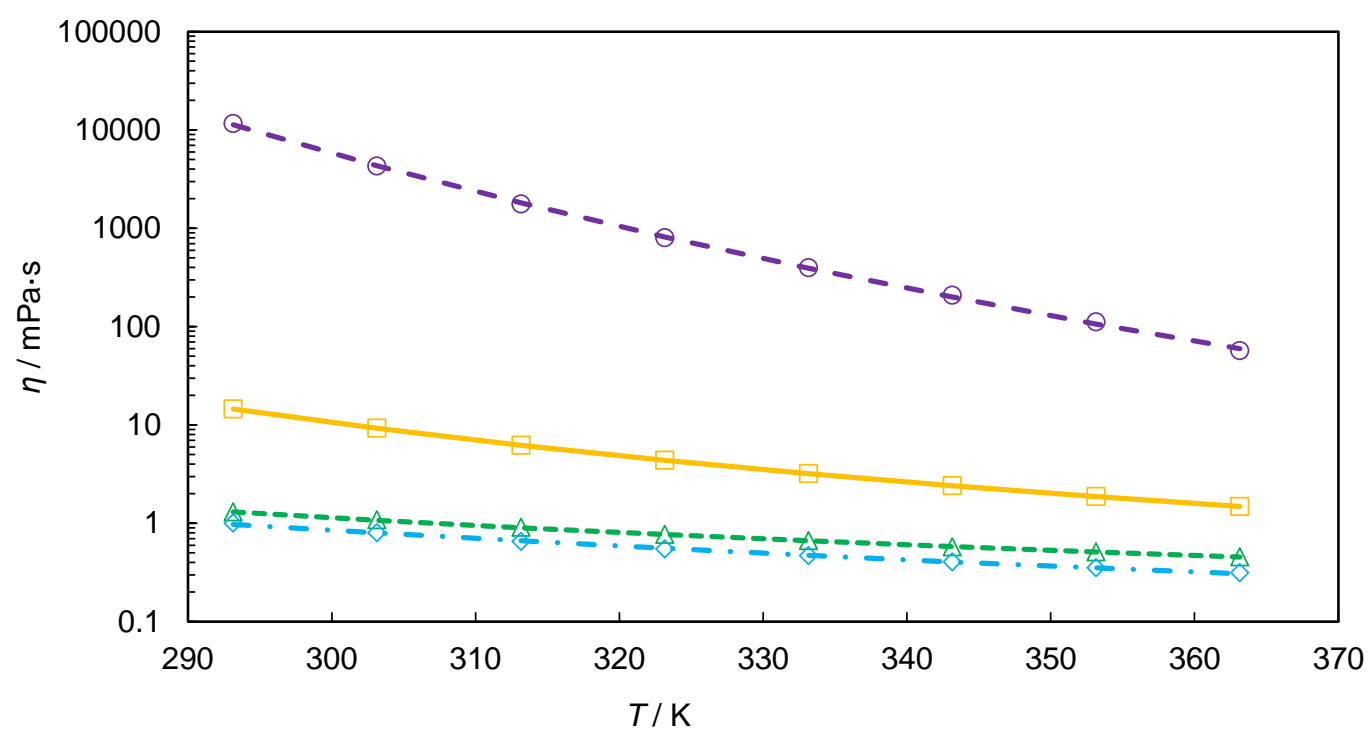

Figure S5. Experimental and calculated viscosities of pure components. Marks and lines: experimental and calculated data. Explanation: EAE ( $\square$ and solid line), DEGDEE ( $\Delta$ and square-dot line), carbamate ( $\circ$ and dashed line), and water $(\diamond$ and dash-dot line).

\section{Density and viscosity modelling using other equations}

Hartono model ${ }^{8,9}$ was also used to correlate our densities. The comparison between experimental and calculated density using this model is plotted in Figure S6. The AARDs are listed in Table S13. It can say that Hartono equation also made a good calculation for our calibration solutions. However, for quaternary solutions, the NRTL-DVOL equation gave a better result. 


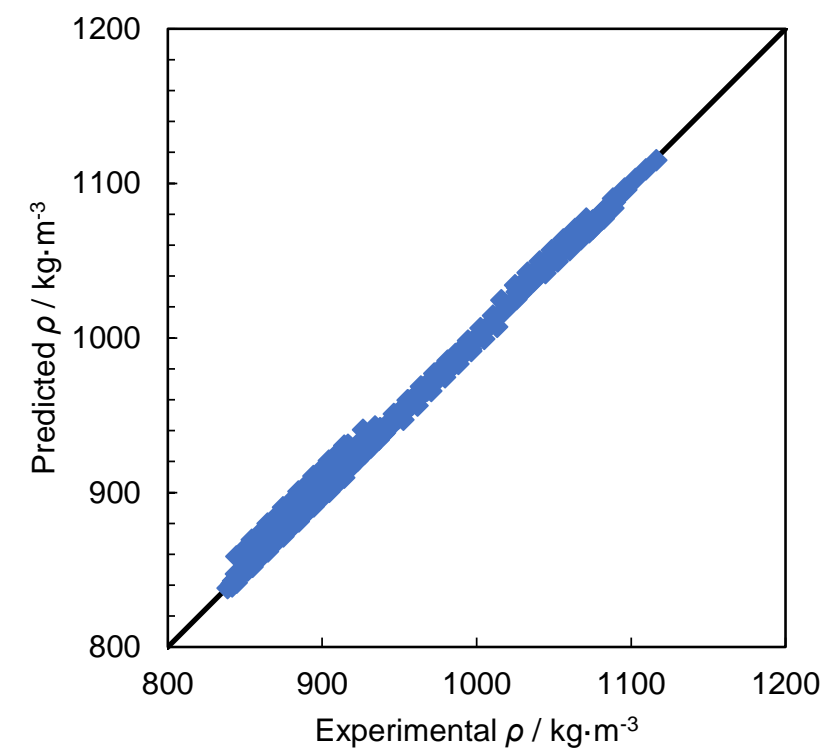

Figure S6. Comparison between experimental and calculated densities for all systems using Hartono equation.

Table S13. AARDs and number of data (n) in density modelling using Hartono equation for EAE/DEGDEE/water/carbamate mixtures.

\begin{tabular}{lcc} 
& $\mathrm{n}$ & $\begin{array}{c}\text { AARD } \\
{[\%]}\end{array}$ \\
\hline EAE/DEGDEE/water & 131 & 0.05 \\
EAE/water/carbamate & 96 & 0.08 \\
EAE/DEGDEE/carbamate & 108 & 0.12 \\
EAE/DEGDEE/water/carbamate & 193 & 0.60
\end{tabular}

For viscosity, the Grunberg-Nissan equation ${ }^{1,2,10}$ was also applied to our data. (Figure S7).

However, the large deviation between experimental and calculated viscosity reinforces the 
unsuitable of this equation to water-containing solutions. The AARDs are presented in Table S14.

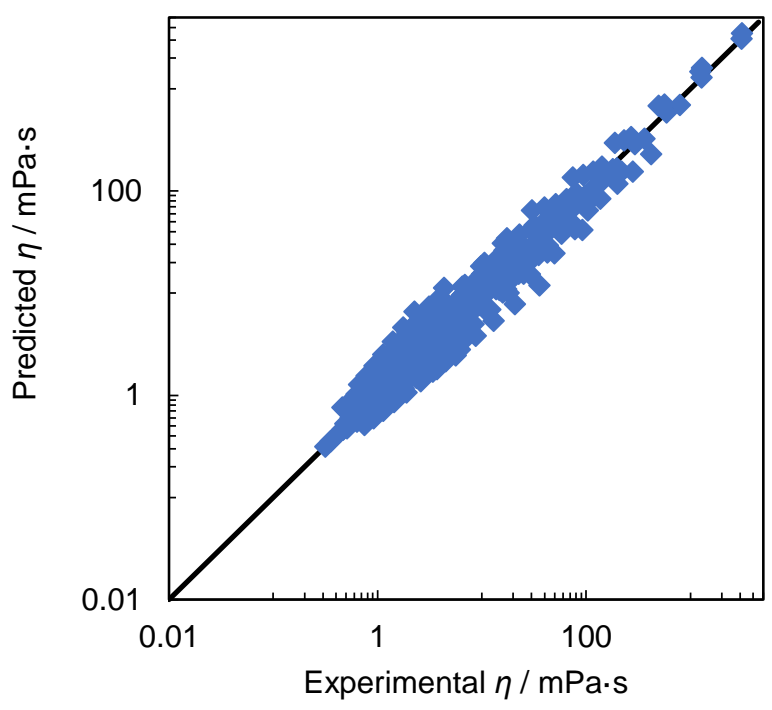

Figure S7. Comparison between experimental and calculated viscosities for all systems using Grunberg-Nissan equation. 
Table S14. AARDs and number of data (n) in viscosity modelling using Grunberg-Nissan equation for EAE/DEGDEE/water/carbamate mixtures

\begin{tabular}{lcc} 
& $\mathrm{n}$ & $\begin{array}{c}\text { AARD } \\
{[\%]}\end{array}$ \\
\hline EAE/DEGDEE/water & 131 & 19.08 \\
EAE/water/carbamate & 112 & 24.08 \\
EAE/DEGDEE/carbamate & 108 & 5.46 \\
EAE/DEGDEE/water/carbamate & 193 & 40.58
\end{tabular}


References

(1) García-Abuín, A.; Gómez-Díaz, D.; Navaza, J. M.; Rumbo, A.; Yaşarołlu, Ö.

Density, Speed of Sound, Viscosity, and Excess Properties of n -Ethyl-2-Pyrrolidone + 2-

(Methylamino)Ethanol [or 2-(Ethylamino)Ethanol] from $\mathrm{T}=(293.15$ to 323.15$) \mathrm{K} . J$. Chem.

Eng. Data 2015, 60, 795-800.

(2) Álvarez, E.; Gómez-Díaz, D.; La Rubia, M. D.; Navaza, J. M. Densities and Viscosities of Aqueous Ternary Mixtures of 2-(Methylamino)Ethanol and 2(Ethylamino)Ethanol with Diethanolamine, Triethanolamine, N-Methyldiethanolamine, or 2-Amino-1-Methyl-1-Propanol from 298.15 to 323.15 K. J. Chem. Eng. Data 2006, 51.

(3) Chowdhury, F. I.; Akhtar, S.; Saleh, M. A.; Khandaker, M. U.; Amin, Y. M.; Arof, A. K. Volumetric and Viscometric Properties of Aqueous Solutions of Some Monoalkanolamines. J. Mol. Liq. 2016, 223, 299-314.

(4) Ku, H. C.; Tu, C. H. Densities and Viscosities of Seven Glycol Ethers from 288.15 K to 343.15 K. J. Chem. Eng. Data 2000, 45, 391-394. 
(5) Esteve, X.; Conesa, A.; Coronas, A. Liquid Densities, Kinematic Viscosities, and Heat Capacities of Some Alkylene Glycol Dialkyl Ethers. J. Chem. Eng. Data 2003, 48, 392397.

(6) Shokouhi, M.; Jalili, A. H.; Samani, F.; Hosseini-Jenab, M. Experimental Investigation of the Density and Viscosity of $\mathrm{CO}_{2}$-Loaded Aqueous Alkanolamine Solutions. Fluid Phase Equilib. 2015, 404.

(7) Yamaguchi, T.; Ando, R.; Yoshida, K.; Yamaguchi, T.; Machida, H. Shear Viscosity and Heterogeneous Structure of Alkylaminoethanol-Based $\mathrm{CO}_{2}$ Absorbents. $J$. Phys. Chem. B 2018, 122, 4045-4050.

(8) Hartono, A.; Mba, E. O.; Svendsen, H. F. Prediction of $\mathrm{N}_{2} \mathrm{O}$ Solubility in Alkanolamine Solutions from the Excess Volume Property. Energy Procedia 2013, 37, $1744-1750$.

(9) Hartono, A.; Mba, E. O.; Svendsen, H. F. Physical Properties of Partially $\mathrm{CO}_{2}$ Loaded Aqueous Monoethanolamine (MEA). J. Chem. Eng. Data 2014, 59, 1808-1816. 
(10) Mandal, B. P.; Kundu, M.; Bandyopadhyay, S. S. Density and Viscosity of Aqueous Solutions of (N-Methyldiethanolamine + Monoethanolamine), (N-Methyldiethanolamine + Diethanolamine), (2-Amino-2-Methyl-1-Propanol + Monoethanolamine), and (2-Amino-2Methyl-1-Propanol + Diethanolamine). J. Chem. Eng. Data 2003, 48, 703-707. 